\title{
Voltage noise and surface current fluctuations in the superconducting surface sheath
}

\author{
J. Scola, ${ }^{1}$ A. Pautrat, ${ }^{1}$ C. Goupil,${ }^{1}$ L. Méchin, ${ }^{2}$ V. Hardy, ${ }^{1}$ and Ch. Simon ${ }^{1}$ \\ ${ }^{1}$ CRISMAT/ENSI-Caen, UMR 6508 du CNRS,6 Bd Marechal Juin, 14050 Caen, France. \\ ${ }^{2}$ GREYC/ENSI-Caen, UMR 6072 du CNRS, 6 Bd Marechal Juin, 14050 Caen, France.
}

\begin{abstract}
We report the first measurements of the voltage noise in the surface superconductivity state of a type-II superconductor. We present strong evidences that surface vortices generates surface current fluctuations whose magnitude can be modified by the pinning ability of the surface. Simple twostage mechanism governed by current conservation appears to describe the data. We conclude that large voltage fluctuations induced by surface vortices exist while the bulk is metallic. Furthermore, this experiment shows that sole surface current fluctuations can account for the noise observed even in the presence of vortices in the bulk.
\end{abstract}

PACS numbers: $74.40 .+\mathrm{k}, 74.25 . \mathrm{Op}, 72.70 .+\mathrm{m}, 74.70 . \mathrm{Ad}$

Separating bulk and surface effects is an ubiquitous problem in condensed matter physics. For example, Flicker noise in semi-conductors [1] and $1 / f$ like-noise in metals 2, 3] can occur from either bulk or surface localized sources. Similarly, one can cite the long standing debate about surface versus bulk effects in driven nonlinear systems like charge density waves [4] or vortex lattice in superconductors [5, 6]. Such debate extends to the origin of the fluctuations responsible for their electronic noise. In any case, the key question is how to determine the relevant sources of disorder. From a technological point of view, noise is a limiting factor for most of applications, and it is necessary to know its origin before expecting its minimization. It is also of theoretical interest to know if, when analyzing disordered systems, boundaries effects are of first importance or if they can be neglected compared to the pure bulk treatment of the problem. A similar question has lead to the proposition that bulk impurities play no major role in the broad band noise generation of charge density waves [7]. As a model system, the case of superconducting vortex lattices can be particularly instructing.

The dynamics of a bulk vortex lattice has been heavily studied in the literature. It is both understood and experimentally shown that the vortices, when submitted to an overcritical current $I>I_{c}$ and under steady state conditions, move in the bulk of the sample with a well defined average periodicity [8]. This motion is associated with a resistance $R_{f f} \leq R_{n}$ and an electrostatic field $E=-V_{L} \wedge \omega\left(V_{L}\right.$ is the line velocity and $\omega$ the vortex field) 9]. Experimentally, it was also shown that $E$ can be separated into its mean $\langle E\rangle$ and its fluctuating part $\delta E^{*}[10$. The latter is the electric field noise. Combined with the V-I relation $V=R_{f f}\left(I-I_{c}\right)$, one can easily realize that this noise can be expressed as fluctuations in the bulk current $\left(I-I_{c}\right)$, or as fluctuations in the resistance $R_{f f}$. Most of the theories invoke bulk pinning centers through their interaction with the moving vortices, leading to vortex density fluctuations and to their associated $\delta R_{f f}^{*}$ fluctuations [10]. Alternatively, noise may arise from over-critical current fluctuations $\delta\left(I-I_{c}\right)^{*}$, while $R_{f f}$ is constant 12]. For the simple reason of current conservation, $\delta\left(I-I_{c}\right)^{*}=\delta I_{c}^{*}$. The fluctuations are thus linked to the underlying pinning mechanism. Crosscorrelation magnetic flux noise experiments and rigorous analysis of the correlation terms are in favor of a surface origin, at least in $\mathrm{Pb}-\mathrm{In}$ samples 12. If fluctuations are pure surface currents, it should be realized that the bulk is only a host which transports the (here noisy) information.

As a consequence, the study of pure surface current fluctuations coexisting with a "noise-free" (metallic) bulk should bring strong experimental evidences on the validity or irrelevancy of this scenario. In the surface superconductivity state, surface currents naturally coexist with a metallic bulk. This means that the $\delta R_{f f}^{*}$ term should be negligible above $B_{c 2}$, so that only current fluctuations can account for the possible voltage noise. To our knowledge, the noise in the surface superconductivity state has not been reported so far. We show here that large voltage fluctuations do exist in this regime. Since it is made clear that they originate from the surface (approximately $0.01 \%$ of the total volume), a demonstration of the relevance of the two-stage surface/bulk noise mechanism is brought. These fluctuations exhibit the same magnitude as it is observed in the conventional vortex state of type II superconductors and can be modeled by similar arguments of statistical averaging.

The prediction of Saint James and De Gennes 13 that superconductivity persists in a surface sheath up to a field $B c_{3} \approx 1.69 B c_{2}$ has been confirmed by many experiments. In particular, surface superconductivity is very frequently observed in low kappa superconducting metals. Consequently, a strip of pure Niobium $\left(T_{c}=9.17 \mathrm{~K}\right.$, GinzburgLandau parameter $\kappa \approx 1$, coherence length $\xi \approx 30 \mathrm{~nm}$ ) [14] has been chosen for this experiment. For all measurements, the magnetic field $\mu_{0} B$ was applied perpendicular to the large faces. Note that, strictly speaking, superconductivity nucleates on the typical scale of $\xi$ over surfaces parallel to $\mu_{0} B$. In practice, this condition is locally realized by non-zero normal component thanks to usual surface roughness, explaining the possi- 
bility of important surface superconducting currents close to the largest surfaces. Kulik calculated that this surface sheath is populated by quantized flux spots ("Kulik's vortices" 15]). It is very likely that the local surface disorder acts as the pinning potential for this short vortices (16] and references herein), leading to a surface critical state. Therefore, the associated superficial critical current $i_{c}(\mathrm{~A} / \mathrm{m})$ comes from a pinning mechanism, and is consequently observed lower than its theoretical upper bound [17]. For $I \geq I_{c} \approx 2 W \cdot i_{c}$ (W=width), surface vortex depinning occurs. Any excess current $\left(I-I_{c}\right)$ is transported in the bulk by normal electrons. It is important to realize that the associated transport equation $V=R_{n}\left(I-I_{c}\right)=R_{n} I_{b u l k}$ implies that $I_{c}$ does not participate in the dc voltage response. In other words, the flux spots move in the surface sheath while being in their critical state most of the time.

This dynamic behavior corresponds to the mean motion and explains the main dc properties. In addition to this mean-field like picture, one can speculate that, during the flux spots motion, many instabilities occur close the surface due to the local release of boundary conditions [12]. The surface current is time-dependent and can be described by its mean value $i_{c}$ and its standard deviation $u^{*}$. The latter represents the statistical fluctuation's magnitude. One expects large fluctuations $\left(u^{*} \lesssim i_{c}\right)$, correlated in a typical size $c$ larger than the intervortex distance $a_{0}$. The scale of the measurement is given by $S$, the surface contained between the contacts. $N=S / c^{2}$ statistically independent fluctuators have to be considered, and their statistical averaging reduces the apparent fluctuations to $\delta i_{c}^{*}=u^{*} / \sqrt{N}$. Up to now, only the surface current flowing in a very thin layer (about $\xi \approx 30 \mathrm{~nm}$ compared to a sample thickness of $\left.10^{4} \times \xi\right)$ is the fluctuating quantity. The measured voltage noise is $\delta V^{*}=R_{n} \delta I_{b u l k}^{*}$. Now the current conservation implies that surface current fluctuations are counterbalanced by bulk current fluctuations $\delta I_{b u l k}^{*}=\delta I_{c}^{*} \approx 2 W \delta i_{c}^{*}$. Finally, the rms noise is given by:

$$
\delta V^{*}=R_{n} \delta I_{c}^{*}=R_{n} I_{c} \sqrt{c^{2} / S}
$$

Experimental signatures of this mechanism would be the existence of large voltage noise even without bulk vortices, i.e., in the surface superconducting regime, and the verification this noise is controlled by the surface critical current.

One of the most critical points in this experiment is to identify properly the surface superconducting regime. Several experimental techniques were performed for this purpose (fig. 1). Specific heat measurements give a robust localization of the second critical field, i.e., the field below which bulk superconductivity appears. Under liquid Helium temperature $(\mathrm{T}=4.2 \mathrm{~K})$, we find that the onset of the bulk superconducting signal is at $B c_{2}=$ $0.295 \pm 0.005 T$ (fig. 1 top), in good agreement with value representative of pure Niobium [14]. The magnetization curve shows the existence of small hysteretic currents $i_{c}$ above $B c_{2}$ (fig. 1 middle), which are characteristics a surface superconducting state 18. All the departure from the Ohmic normal state behavior is due to these surface currents [19]. At the same time, the differential resistance $R$ is $d V / d I \approx R_{n}$ at high current even if the normal state is not reached, as simply state by $V / I \neq R_{n}$ (fig. 1 bottom). Such a small difference between $R$ and $R_{n}$ can naturally be attributed to the smallness of the surface sheath volume, where the superconducting order parameter can relax. $B c_{3}$ can be estimated from the disappearance of $i_{c}$, here at $0.52 \pm 0.02 \mathrm{~T}$. The obtained ratio $B c_{3} / B c_{2}=1.76 \pm 0.06$ is close to the theoretical value 1.69. Since the critical region where surface superconductivity takes place has been delimited, we can investigate if an excess noise exists in this regime and eventually analyze its behavior.

The principle of the conduction noise measurements is the following one. The temporal evolution of the voltage is digitally acquired after ultra low noise differential amplification (NF-SA 400F3) with usual care to eliminate external interference 20] (Fig. 2). It is then Fourier Transformed and squared in order to obtain the Power Spectral Density of the voltage fluctuations $\tilde{V}^{2}(f)$. The final resolution at low frequency is 0.7 $\mathrm{nV} / \sqrt{H z}$. Shown in the figure 3 (Top) is the variation of $\delta V^{*}=\sqrt{\int \tilde{V}^{2}(f) d f}$, integrated over the low frequency bandwidth $(6 \mathrm{~Hz}-200 \mathrm{~Hz})$, as function of the magnetic field. For $B \geq B_{c 3} \approx 0.52 T$, no measurable noise is observed, meaning that the metallic part (and the contacts) of the sample can be considered as noise-free within our resolution. This confirms that $R_{n}$ is not a relevant fluctuating quantity in this experiment. For $B \lesssim B_{c 3}$, the noise appears and grows when the field is decreased. Also shown in figure 3 (Bottom) is the variation of the surface critical current $i_{c}(A / \mathrm{cm})$ measured by V-I characteristics. In viewing the data, it is clear that both $\delta V^{*}$ and $i_{c}$ have a similar field dependance. This suggests a simple proportionality between $i_{c}$ and the amount of voltage noise. This is expected from equation (1) in the simplest case where $c$ is a constant, and a numerical application gives $c \approx 0.8 \pm 0.2 \mu m$. It is important to note that a similar analysis leads to $c \lesssim 1 \mu m$ in the bulk vortex state of conventional type II superconductors [12, 20]. The voltage noise in the surface superconducting state appears to have the same magnitude as in the conventional mixed state. The typical variation of $\delta V^{*}$ versus the transport current is shown in figure 4 . This closely mimics the noise variation during the depinning transition a bulk vortex lattice [10]. Thus, as proposed in 12, 20], there should be a general explanation of the moving vortex noise in terms of pure surface current fluctuations.

In order to confirm the role of the large surfaces for the noise generation, we have modified the top and bottom surfaces properties using a low energy ion irradiation 
$\left(A r^{+}\right.$ions with a kinetic energy of $600 \mathrm{eV}$ and $30 \mathrm{~min}-$ utes exposure). Because of the short range of the ions, the damage is restricted in the first nm of the sub-surface region. Bulk properties $\left(\rho_{n}, B_{c 2}, T_{c}\right)$ are consistently measured as unchanged. The genuine irradiation effect on the surface superconductivity properties can be a matter of debate. AFM and MEB inspections confirm that the surface topography exhibits a strong increase of its roughness at low spatial scale [21]. The increase of the surface roughness results in stronger flux spots pinning ability, but at the same time the strength of the surface sheath is reinforced because the roughening process multiplies the possibilities that superconductivity nucleates at the scale of the coherence length $(\xi \approx 30 \mathrm{~nm})$. Furthermore, as shown in figure 4 , the third critical field has changed and increased up to at least $0.6 T$, likely because of the concentration of impurities (implying a reduced electron mean free path) near the surface. Whatever the genuine reason is, the central point is that an impressive increase of the surface critical current is observed (fig. 5 ), whereas the bulk properties are unchanged. Interestingly for applications, one obtains large critical currents values $\left(i_{c} \approx 10 \mathrm{~A} / \mathrm{cm}\right.$ or $J_{c} \approx 8.10^{4} \mathrm{~A} / \mathrm{cm} 2$ if expressed like a current density at the sample scale) for fields much higher than $B c_{2}$. As shown in figure 6 , an associated increase of the noise is observed, giving more weight to the evidence of its pure surface origin. Furthermore, quantitative analysis yields $c=1 \pm 0.2 \mu \mathrm{m}$ after the irradiation, very close to the fluctuator size found for the virgin sample. This means that almost all the increase of noise comes from the increase of the current fluctuation magnitude, and that this latter is given by the critical current value. A more detailed discussion of the effect of surface irradiation for the pinning and vortex noise in the surface superconducting regime and in the mixed state ( $B \leq B c_{2}$ ), including the statistical study of the noise spectra, will be discussed widely elsewhere.

To conclude, voltage noise due to flux spots motion in the superconducting surface sheath has been observed. Current conservation induces bulk noisy current whereas the noisy sources were shown to be clearly localized close to the surface. Noise is found to be of the same magnitude as in the conventional mixed state of type II superconductors, and it behaves similarly. This emphasizes the fundamental role of the boundaries in the non-linear response of vortices.

Acknowledgments: This experiment was supported by "la région basse Normandie".

[1] A. Van Der Ziel, fluctuations phenomena in semiconductors, Edited by C.A. Hogarth, London, Butterworths scientific publications (1959).

[2] P. Dutta and M. Horn, Rev. Mod. Phys. 53, 497 (1981).
[3] N. M. Zimmerman, J. H. Scofield, J. V. Mantese, and W. W. Webb, Phys. Rev. B 34, 773 (1986).

[4] G. Grüner, Rev. Mod. Phys. 60, 1129 (1988).

[5] Y. Paltiel, E. Zeldov, Y. Myasoedov, M. L. Rappaport, G. Jung, S. Bhattacharya, M. J. Higgins, Z. L. Xiao, E. Y. Andrei, P. L. Gammel, and D. J. Bishop Phys. Rev. Lett. 85, 3712 (2000).

[6] N. Lütke-Entrup, B. Plaçais, P. Mathieu, and Y. Simon Phys. Rev. Lett. 79, 2538-2541 (1997).

[7] N. P. Ong, G. Verma, and K. Maki, Phys. Rev. Lett. 52, 663 (1984).

[8] E. M. Forgan, P. G. Kealey, S. T. Johnson, A. Pautrat, Ch. Simon, S. L. Lee, C. M. Aegerter, R. Cubitt, B. Farago, and P. Schleger Phys. Rev. Lett. 85, 3488 (2000).

[9] B. D. Josephson, Phys. Lett. 16, 242 (1965).

[10] J. R. Clem, Phys. Rep. 75, 1 (1981).

[11] Y. Paltiel, G. Jung, Y. Myasoedov, M. L. Rappaport, E. Zeldov, S. Bhattacharya, and M. J. Higgins, Fluctuation and Noise Lett. 2, 31 (2002).

[12] B. Plaçais, P. Mathieu, and Y. Simon, Phys. Rev. Lett. 70, 1521 (1993).

[13] D. Saint James and P.G. De Gennes, Phys. Lett. 7, 306 (1964).

[14] W. DeSorbo, Phys. Rev. 135, A1190 (1965).

[15] I.O. Kulik, Sov. Phys. JETP 28, 461 (1969).

[16] P. Mathieu, B. Plaçais and Y. Simon, Phys. Rev. B 48, 7376 (1993).

[17] H.R. Hart, Jr. and P.S. Swartz, Phys. Rev. 156, 403 (1966).

[18] H. J. Fink and L. J. Barnes, Phys. Rev. Lett. 15, 792 (1965).

[19] C. F. Hempstead and Y. B. Kim, Phys. Rev. Lett. 12, 145 (1964).

[20] J. Scola, A. Pautrat, C. Goupil and Ch. Simon, Phys. Rev. B 71, 104507 (2005).

[21] The roughness has been controlled by AFM measurements. We have found $0.7 \mathrm{~nm} \mathrm{rms}$ before the irradiation and $2.2 \mathrm{~nm}$ rms after the irradiation (measurements averaged over a length of $1 \mu \mathrm{m})$. 


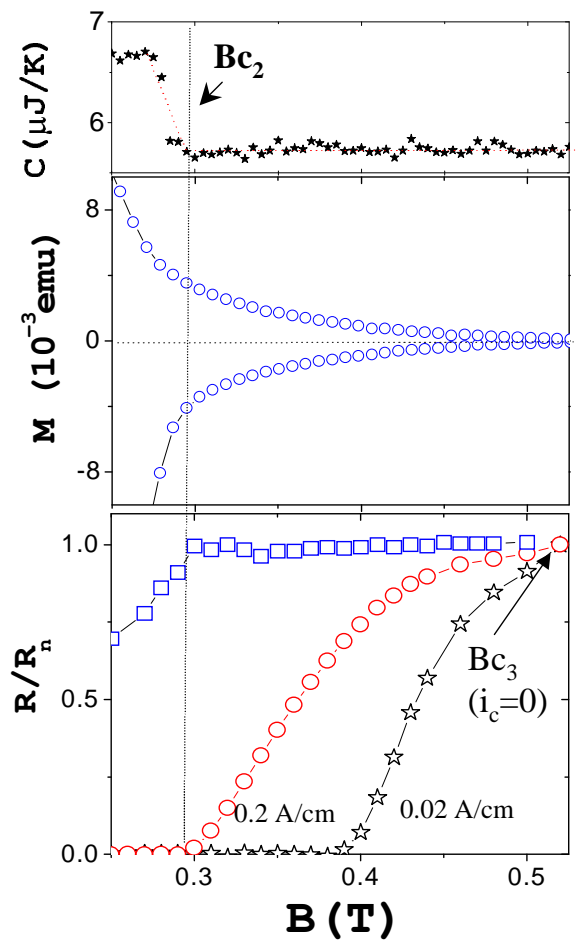

FIG. 1: Evidence of surface superconductivity at $\mathrm{T}=4.2 \mathrm{~K}$ in the Niobium strip). Top: Specific heat as function of B. Middle: Magnetization measured by a SQUID magnetometer. Note the hysteresis above $B c_{2}$. Bottom: Resistance normalized by its normal state value. (stars: $i=0.02 \mathrm{~A} / \mathrm{cm}$, circles: $i=0.2 \mathrm{~A} / \mathrm{cm}$, squares: differential resistance for $i \gg i_{c}$ ). The non-Ohmic behavior is due to the surface critical current.

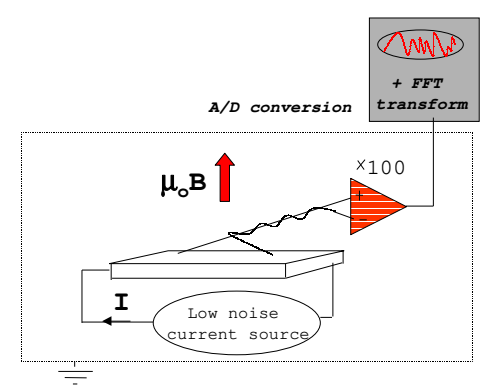

FIG. 2: Experimental set-up used to measure the voltage noise in a Niobium strip ( $\left.\operatorname{size} \mathrm{L}=5^{*} \mathrm{~W}=1.5^{*} \mathrm{t}=0.2 \mathrm{~mm}^{3}\right)$. 


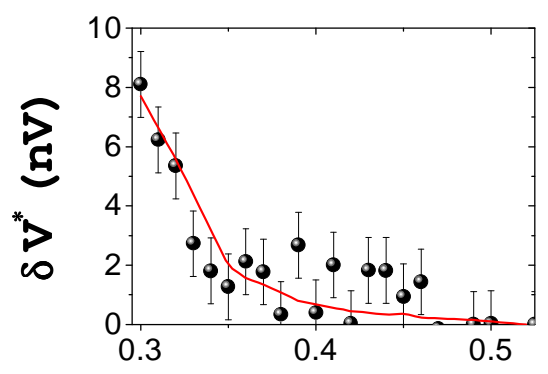

B (T)

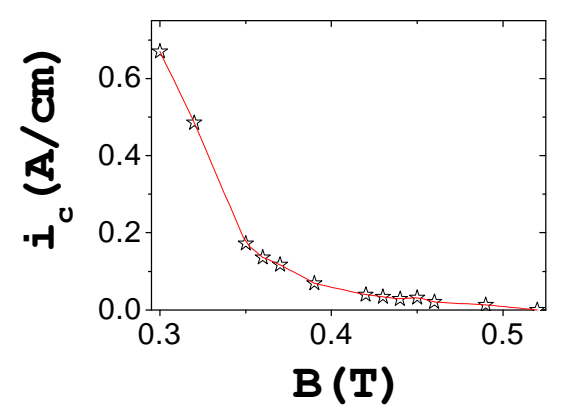

FIG. 3: Top: Variation of the voltage noise measured in the quasi-linear regime of flux spots motion for $B c_{2} \leq B \leq B c_{3}$ $(\mathrm{T}=4.2 \mathrm{~K})$. Also shown the master curve corresponding to the variation of $i_{c}(B)$. Bottom: Variation of the surface critical current for $B c_{2} \leq B \leq B c_{3}(\mathrm{~T}=4.2 \mathrm{~K})$. The dotted line is a guide for the eyes.

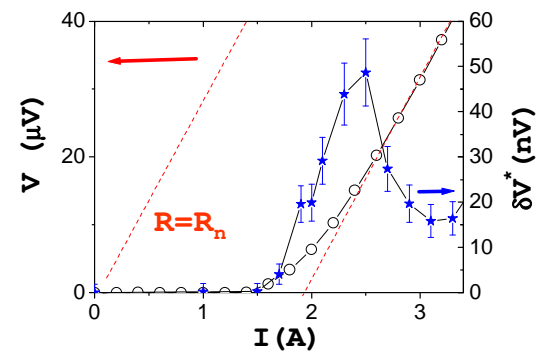

FIG. 4: The voltage and the voltage noise as function of the current $(\mathrm{T}=4.2 \mathrm{~K}, \mathrm{~B}=0.36 \mathrm{~T}$, irradiated surfaces). Note the depinning peak followed by the more quiet behavior characteristic of a depinning transition. The flux-flow noise (see fig. 5) corresponds to the regime after the depinning peak. This corresponds to the steady state of the motion. 


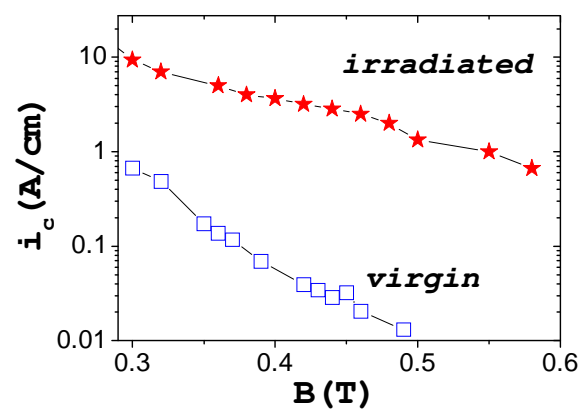

FIG. 5: Surface critical current for $B c_{2} \leq B \leq B c_{3}$ for the virgin and for the irradiated samples $(\mathrm{T}=4.2 \mathrm{~K})$. Note the huge increase of $i_{c}$ after the surface irradiation.

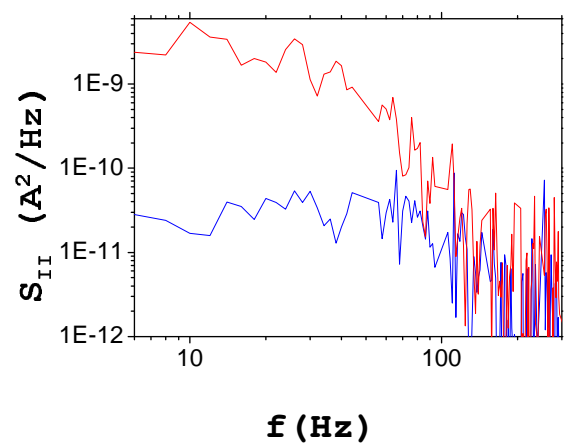

FIG. 6: Typical excess of flux flow noise (the units correspond to the spectral density of the elementary surface current fluctuations $\left.S_{I I}=S_{v v} . S /\left(2 . W . R_{n}\right) 2\right)(\mathrm{T}=4.2 \mathrm{~K}, \mathrm{~B}=0.36 \mathrm{~T})$. Top curve: irradiated sample. Bottom curve: virgin sample. The integration of these spectra over the $6 \mathrm{~Hz}-200 \mathrm{~Hz}$ bandwidth gives $i_{c} . c$, the value of the elementary fluctuation (in Amperes). $c$ is deduced from this value. 